\title{
Die Gestaltung des Übergangs zur Weiterführenden Schule - Welche Maßnahmen wurden und werden an Grundschulen in NRW praktiziert?
}

\author{
Stefanie van Ophuysen (iD - Sina Schürer $(D)$ Bea Bloh
}

Eingegangen: 1. Juni 2020 / Angenommen: 11. Oktober 2020 / Online publiziert: 13. Januar 2021

(C) Der/die Autor(en) 2021

Zusammenfassung Studien zum Übergang von der Grund- in die weiterführende Schule gibt es zu zahlreichen Themen. Wie Grundschulen den Übergang zur weiterführenden Schule gestalten, wurde bislang jedoch kaum erforscht. Auf Basis des Forschungsstands werden relevante Maßnahmen identifiziert. In einer online-Befragung an 106 Grundschulen in Nordrhein-Westfalen wurde erfasst, inwiefern diese Maßnahmen tatsächlich umgesetzt werden und als wie relevant bzw. schwierig ihre Umsetzung erachtet wird. Die Ergebnisse werden verglichen mit Daten einer Studie, die 2002 an 71 Grundschulen durchgeführt wurde. Es zeigt sich eine hohe Stabilität der Gestaltung in den letzten 15 Jahren. Maßnahmen, die auf stufenübergreifender Kooperation basieren, werden heute zwar häufiger praktiziert, stehen aber weiterhin am Ende der Rangreihe.

Schlüsselwörter Grundschulübergang · Übergangsgestaltung · Längsschnitt · Stufenübergreifende Lehrerkooperation

Prof. Dr. S. van Ophuysen $(\bowtie) \cdot$ Dr. S. Schürer

Institut für Erziehungswissenschaft, WWU Münster, Georgskommende 33, 48143 Münster,

Deutschland

E-Mail: vanOphuysen@uni-muenster.de

Dr. S. Schürer

E-Mail: schuerer@uni-muenster.de

Prof. Dr. B. Bloh

Institut für Erziehungswissenschaft, Universität Paderborn, Warburger Str. 100, 33098 Paderborn, Deutschland

E-Mail: bea.bloh@uni-paderborn.de 


\title{
Managing the transition to secondary school-What measures have been and are being practised at primary schools in NRW?
}

\begin{abstract}
Studies on the transition from primary to secondary school are available on numerous topics. However, little research has been done on how primary schools manage the transition to secondary school. Relevant measures are identified on the basis of the state of research. An online survey of 106 primary schools in North Rhine-Westphalia was conducted to determine the extent to which these measures are actually implemented and how important or difficult their implementation is considered. The results are compared with data from a study carried out at 71 primary schools in 2002. It shows a high stability of design over the last 15 years. Although measures based on cooperation across school levels are more common today, they are still at the bottom of the ranking.
\end{abstract}

Keywords Primary school transition - Transition design · Longitudinal study • Cross level teacher cooperation

\section{Einleitung}

Jährlich wechseln rund 725.000 Kinder von der Grundschule zur weiterführenden Schule, allein im größten Bundesland Nordrhein-Westfalen waren es im Schuljahr 2018/2019 155.114 Kinder (Ministerium für Schule und Bildung des Landes Nordrhein-Westfalen 2019). Mit dem Übertritt wird für sie eine Entscheidung getroffen, die mittelfristig für die weitere Lern- und Leistungsentwicklung und damit letztlich auch für Karrierechancen und den beruflichen Werdegang prädiktiv ist (Autorengruppe Bildungsberichterstattung 2016; Maaz et al. 2008). Andererseits ist der Übergang auch für das unmittelbare Erleben der Schülerinnen und Schüler relevant, da er eine potenziell emotional belastende Phase darstellt, deren Bewältigung für das (schulische) Wohlbefinden bedeutsam ist (Kurtz et al. 2010; Leffelsend und Harazd 2004; Sirsch 2000; van Ophuysen 2018). Die Relevanz des Übergangs und seiner Gestaltung steht damit außer Frage.

Da die aktuelle Praxis der Übergangsgestaltung jedoch bislang allenfalls punktuell erforscht ist (Porsch 2015), möchten wir mit diesem Beitrag einen ersten Überblick zu der Frage liefern, welche Maßnahmen in den Grundschulen praktiziert und als bedeutsam erachtet werden. Dabei fokussieren wir auf Maßnahmenbereiche, die auf Basis theoretischer und empirischer Erkenntnisse als relevant für einen gelingenden Übergang gelten können.

\section{Ableitung von Gestaltungsmaßnahmen}

\subsection{Identifikation relevanter Maßnahmenbereiche}

Das integrative Rahmenmodell der Übergangsqualität (van Ophuysen und Harazd 2014) identifiziert Merkmale, die über das Gelingen des Grundschulübergangs Aus- 


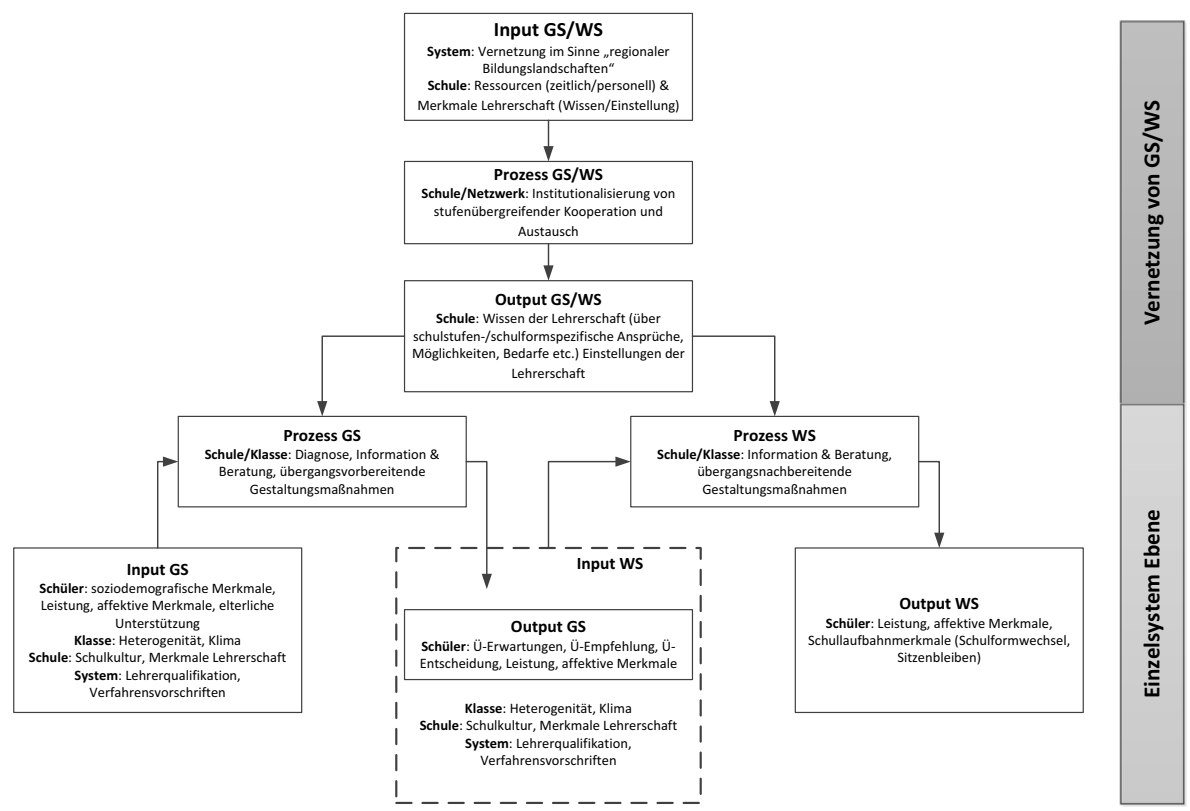

Abb. 1 Rahmenmodell der Übergangsqualität (van Ophuysen und Harazd 2014)

kunft geben. In Anlehnung an klassische Modelle der Schulqualität (z. B. Scheerens 1990; Ditton 2000) wird zwischen Qualitätsindikatoren auf Input-, Prozess- und Output-Ebene unterschieden. Um der besonderen Situation des Übergangs gerecht zu werden, werden Grund- und weiterführende Schule einerseits als separate Gestaltungseinheiten (Fend 1986; Dalin et al. 1995) betrachtet, die nacheinander das Übergangsgeschehen prägen. Dabei übernimmt die Grundschule vornehmlich die Verantwortung für die Vorbereitung des Schulwechsels, während die weiterführende Schule darauf aufbauend die Bewältigung des Neuanfangs unterstützt. Darüber hinaus berücksichtigt das Modell aber auch den systemischen Aspekt, dass ein qualitätsvoller Übergang nur in gemeinsamer Verantwortung beider Schulen gelingen kann und daher der stufenübergreifenden Vernetzung und Kooperation bedarf. Für die vorliegende Arbeit betrachten wir diejenigen Bausteine des Modells, an denen die Grundschule beteiligt ist.

Grundsätzlich gelten die Entfaltung des individuellen Leistungspotentials und die Sicherung des schulischen Wohlbefindens als wichtige Zielvariable von Schule (Hagenauer 2011). Seitens der Grundschule kann die Vorbereitung auf den Übergang entsprechend dann als gelungen gelten, wenn die Schülerinnen und Schüler in ihrem fachlichen und sozialen Lernen gut auf die anstehenden Herausforderungen vorbereitet sind, und wenn sie mit positiven Erwartungen auf den Wechsel blicken. In der Verantwortung der Grundschule liegt es weiterhin, eine Übergangsempfehlung zu erteilen, die den Potenzialen des individuellen Kindes angemessen ist und durch die elterliche Entscheidung mitgetragen wird (siehe Abb. 1, „Output GS“).

Schulische Maßnahmen, die der Erreichung dieser Ziele dienen, sind im Modell auf der Prozess-Ebene verortet. Sie betreffen die sozio-emotionale Begleitung der 
Kinder und die Vorbereitung auf fachliche, unterrichtsbezogene Veränderungen. Mit Blick auf die Schulformempfehlung sind lehrkraftseitige diagnostische Aktivitäten ebenso erforderlich, wie die Beratung und Informierung der Eltern (van Ophuysen und Harazd 2011; siehe Abb. 1, „Prozess GS“).

Wie gut solche Prozesse gelingen, hängt von der Qualifikation der individuellen Lehrkraft sowie von den institutionellen Unterstützungsmöglichkeiten ab. Daher stellen auch übergangsbezogene Maßnahmen der Personal- und Organisationsentwicklung Indikatoren der Übergangsqualität dar. Sie können innerhalb der Grundschule verortet sein (vgl. Abb. 1, „Input GS“), finden aber ebenso in der Kooperation von Grund- und weiterführender Schule ihren Raum (vgl. Abb. 1, „Prozess GS/WS“).

Im Folgenden werden empirische Befunde berichtet, die innerhalb der drei Bereiche a) fachliche und affektive Unterstützung der Schülerinnen und Schüler, b) Diagnostik und Beratung sowie c) Personal- und Organisationsentwicklung die Ableitung konkreter förderlicher Maßnahmen erlauben.

\subsection{Empirisch begründete Ableitung von Maßnahmen der Übergangsgestaltung}

\subsubsection{Maßnahmen der fachlichen und affektiven Unterstützung der Schülerinnen und Schüler}

Verschiedene nationale und internationale Studien thematisieren das Erleben der Schülerinnen und Schüler vor und nach dem Übergang zur weiterführenden Schule (zusammenfassend van Ophuysen 2018). Deutschsprachige Studien weisen zwar insgesamt auf eine eher positive Grundstimmung hin, machen aber auch auf einen nicht zu unterschätzenden Anteil an Risikokindern aufmerksam, die sich insbesondere durch sozio-emotional ungünstige Ausgangsbedingungen auszeichnen (Büchner und Koch 2001; Leffelsend und Harazd 2003, 2004; Sirsch 2000; van Ophuysen 2006). Bei diesen Kindern ist neben einem eher geringen schulischen Wohlbefinden insbesondere eine hohe Besorgnis zu konstatieren, die sich auf das soziale Miteinander, die organisatorischen Rahmenbedingungen sowie das schulische Lernen und Leisten an der künftigen Schule bezieht (Kurtz et al. 2010; Leffelsend und Harazd 2003; van Ophuysen 2013).

Im Sinne der transaktionalen Stresstheorie (Lazarus und Folkman 1987) kann das Belastungspotential dieser Situation reduziert werden, indem Veränderungen vermieden werden. Die vorbereitende Anpassung an zukünftige curriculare und organisatorische Anforderungen (z.B. Unterricht bei mehreren verschiedenen Fachlehrkräften) ist eine Möglichkeit, die bereits im Vorfeld des eigentlichen Schulwechsels an den Grundschulen genutzt werden kann (van Ophuysen 2013). Doch nicht alle Änderungen sind vermeidbar (neue Mitschülerinnen und Mitschüler, Lehrkräfte, Unterrichtsfächer) und es steht außer Frage, dass neue Situationen auch das Potenzial für positive Erfahrungen und Wachstum beinhalten (Lohaus et al. 2005). Hier gilt es, die Ressourcen der Kinder so zu stärken, dass sie die Herausforderungen als Chance wahrnehmen können (Hildebrandt 2016; Knoppick et al. 2016; Lazarus und Folkman 1987). Schließlich ist das Erleben von Unsicherheit über zukünftige Ereignisse eine weitere stressauslösende Erfahrung. Positiv gewendet bedeutet dies, 
dass durch frühzeitige Informierung über die zu erwartende Situation ein wichtiger Besorgnisfaktor reduziert werden kann (siehe dazu z. B. Schürer et al. 2006).

Die Befunde legen insgesamt nahe, dass eine Übergangsgestaltung, die zu einer positiven Wahrnehmung des Schulwechsels und seiner Chancen beiträgt, durch eine (moderate) Anpassung an Methoden und Routinen der weiterführenden Schule, durch die Stärkung sozialer und fachlicher Ressourcen der Kinder sowie die Informierung über den künftigen Schulkontext geprägt sein sollte.

\subsubsection{Maßnahmen im Rahmen von Diagnostik und Beratung}

Die hohe Relevanz der Übergangsempfehlung/-entscheidung erfordert ein verantwortungsvolles, qualitativ hochwertiges diagnostisches Handeln und entsprechende Kooperation mit den Eltern.

Bisherige Forschungsbefunde stellen die Urteilsqualität der Übergangsempfehlung in Frage. So zeigte sich bereits im Rahmen der ersten großangelegten Schulleistungsstudien nicht nur ein unerwartet starker Zusammenhang zwischen der sozialen Herkunft des Kindes und der Übergangsempfehlung sowie der Übergangsentscheidung (Baumert et al. 2006, 2010), sondern auch eine starke Überlappung der Verteilungen der Testleistungen von Schülerinnen und Schülern an unterschiedlichen weiterführenden Schulformen (z.B. Behörde für Schule und Berufsbildung 2011 (LAU); Bos und Gröhlich 2011 (KESS 8); Jonkmann et al. 2010 sowie Maaz und Nagy 2010 (TIMSS)).

Es erscheint plausibel, dass solche Probleme bei der Erteilung einer Übergangsempfehlung durch einen qualitativ hochwertigen diagnostischen Prozess abgemildert werden können. Beispielsweise stellen die Verfügbarkeit relevanter Informationen und deren Multiperspektivität wichtige Bedingungen für eine gelingende Diagnostik dar (van Ophuysen et al. 2013; Lintorf et al. 2016). Da es sich bei der Übergangsempfehlung um eine Platzierungsentscheidung handelt, sind neben guten Kenntnissen über das individuelle Kind auch Wissen über Merkmale und Anforderungen der umliegenden weiterführenden Schulen bedeutsam. Dies kann insbesondere durch den direkten Kontakt mit weiterführenden Schulen erworben werden.

Ebenso spielen aber auch die an die Eltern gerichteten Beratungs- und Informationsangebote der Grundschule eine wichtige Rolle. Die Eltern sollten über die Leistungen und Potenziale des Kindes einerseits und über die schulischen Angebote andererseits bestmöglich informiert werden (van Ophuysen und Harazd 2014).

Insgesamt lässt sich schlussfolgern, dass sowohl ein hochwertiges diagnostisches Handeln - insbesondere durch die Sammlung und Dokumentation vielfältiger Informationen über die individuellen Kinder unter Einbeziehung der Sichtweise mehrerer Personen (z. B. Eltern, andere Lehrkräfte) und die Informierung über die weiterführenden Schulen, ihre Angebote und Anforderungen - als auch ein eng begleiteter Beratungsprozess der Eltern unter Einbeziehung der Kinder positive Gestaltungsmerkmale des Übergangs sind. 


\subsubsection{Personal- und Organisationsentwicklung}

Damit die oben genannten Maßnahmenbereiche, die im Rahmenmodell der Übergangsqualität auf Ebene des Prozesses der Einzelschule angesiedelt sind, nachhaltig erfolgreich etabliert werden können, müssen sie in Schulentwicklungsprozesse eingebunden werden. Diese sind im Rahmenmodell im Bereich des Inputs auf Systemebene zu verorten. Zum einen gilt es, Strukturen und Abläufe zu institutionalisieren, die der Sicherung der Prozessqualität dienen und diese weitgehend unabhängig vom Engagement individueller Lehrkräfte machen. Zum anderen können die Kompetenzen der Lehrkräfte selbst durch einschlägige Fort- und Weiterbildungen gestärkt werden, um sie auf die anstehenden Herausforderungen angemessen vorzubereiten.

Wie zuvor erwähnt, ist sowohl für die angemessene fachliche und affektive Begleitung der Kinder als auch für eine kompetente Diagnostik und Beratung die Kooperation mit weiterführenden Schulen von Bedeutung. Auch im Rahmenmodell der Übergangsqualität wird insbesondere diese Vernetzung herausgestellt. Im Kontext des Grundschulübergangs hat sich dazu speziell die Idee der (stufenübergreifenden) Schulnetzwerke als zielführend erwiesen, die seit den frühen 2000er Jahren weiterentwickelt und in Projekten erprobt wurde (z. B. SINUS, Schulen im Team, GanzIn). Beispielsweise erlebten Lehrkräfte, die als Netzwerkkoordinator_innen im Projekt Schulen im Team mitarbeiteten, die Arbeit des Schulnetzwerkes insgesamt als sehr gewinnbringend für die Übergangsgestaltung. Dabei wurden insbesondere Vorteile für den Bereich der Elternberatung wahrgenommen, und der Austausch über Fragen des Curriculums und der fachlichen Standards an den beiden Schulstufen wurde positiv wertgeschätzt (Sartory 2016).

Somit stellen auch Maßnahmen der Personal- und Organisationsentwicklung einen wichtigen Bestandteil der Übergangsgestaltung dar, wobei einerseits Weiterbildungsmaßnahmen und andererseits die Verstetigung von Wissen durch Programme/ Dokumentationen und die Institutionalisierung von Kooperationen innerhalb der Schule sowie zwischen Grund- und weiterführenden Schulen bedeutsam zu sein scheinen.

\section{Zielsetzung der Studie}

Durch die Umsetzung entsprechender Maßnahmen der Übergangsgestaltung können Grundschullehrkräfte das Gelingen oder Misslingen des Übergangs mitprägen. Bislang liegen kaum Befunde dazu vor, welche Maßnahmen an Schulen tatsächlich eingesetzt werden (vgl. zusammenfassend Porsch 2018). Daher betrachtet die hier vorliegende Studie den Verbreitungsgrad für eine Auswahl an möglichen Maßnahmen aus den oben abgeleiteten Bereichen, um den Status quo abzubilden. Das sich so ergebende Bild stellt beispielsweise bei der Einordnung von Befunden zum Erleben des Übergangs durch die Schülerinnen und Schüler eine interessante Kontextinformation dar. Zusätzlich wird eruiert, wie schwierig respektive bedeutsam die jeweiligen Maßnahmen von den Lehrkräften eingeschätzt werden. Scheitert beispielsweise die Durchführung von relevant erachteten Maßnahmen an der Schwierigkeit der Umsetzung, so ist die Suche nach und das Aufzeigen von Lösungen ein 
erster Schritt zur Verbesserung der Situation. Die Diskussion um den Grundschulübergang, der zu Beginn der 2000er Jahre relativ intensiv geführt wurde sowie die diesbezüglichen Forschungserkenntnisse der letzten Dekade lassen vermuten, dass diese auch Eingang in die Praxis der Übergangsgestaltung gefunden haben. Daher werden die hier vorliegenden Daten mit den Daten einer analogen Studie aus dem Jahr 2002 (siehe dazu van Ophuysen 2005) verglichen.

Insgesamt dient die vorliegende Studie damit der Klärung folgender Fragestellungen:

1. Wie häufig werden verschiedene Maßnahmen der Übergangsgestaltung aktuell praktiziert?

2. Wie hängen die lehrerseitig wahrgenommene Relevanz und der Verbreitungsgrad (tatsächliche Umsetzung) der Maßnahmen zusammen?

3. Wie gut lässt sich der Verbreitungsgrad der Maßnahmen auf Basis der wahrgenommenen Relevanz der Maßnahme und Schwierigkeit der Umsetzung vorhersagen?

4. Welche Veränderungen sind im Vergleich zur Befragung von 2002 zu erkennen: Welche Maßnahmen werden häufiger, welche seltener praktiziert?

\section{Methode}

\subsection{Durchführung der Befragung}

Die Daten der Studie wurden in den Jahren 2002 und 2018 an Grundschulen in $\mathrm{NRW}^{1}$ erfasst. Da die Durchführung in den beiden Jahren in etwas unterschiedlicher Form erfolgte, werden die Modalitäten für die beiden Jahre separat berichtet.

Im Jahr 2002 wurden 150 Grundschulen in NRW per Zufallsauswahl aus der Liste aller Grundschulen in NRW ausgewählt. Die Schulleitungen wurden postalisch um Teilnahme an der Paper-Pencil-Befragung gebeten. Von diesen beantwortete knapp die Hälfte $(47,3 \%, n=71)$ den Fragebogen für ihre Schule.

Die Erhebung im Jahr 2018 erfolgte als online-Befragung. Per E-Mail wurden 600 zufällig ausgewählte Schulen um Teilnahme gebeten und erhielten den Link zur Befragung. Dies entspricht einem Anteil von $22 \%$ der öffentlichen Grundschulen in NRW (Ministerium für Schule und Bildung des Landes NRW 2019). Zwar blieb der Rücklauf mit nur 17,7\% $(n=106)$ sehr deutlich hinter dem Rücklauf der Vorgängerbefragung, jedoch liegt die Rücklaufquote für eine ,spontane“ Befragung ohne weitere Incentives im zu erwartenden Bereich.

\subsection{Stichprobe}

An beiden Erhebungszeitpunkten zusammen haben 177 Personen stellvertretend für ihre Grundschulen an der Befragung teilgenommen. Der Frauenanteil lag in 2002 bei $70,4 \%$, in 2018 bei 90,6\%. Bei den Befragten handelt es sich insgesamt um

\footnotetext{
1 In NRW stehen Schülerinnen und Schülern nach der Grundschule verschiedene weiterführende Schulformen zur Auswahl: Gymnasium, Realschule, Hauptschule, Gesamtschule und Sekundarschule. Die beiden letztgenannten Schulformen sind auf heterogene Lerngruppen ausgerichtet.
} 
Tab. 1 Schulmerkmale im Vergleich (2002 vs. 2018)

\begin{tabular}{lllllllll}
\hline & $\begin{array}{l}2002 \\
\text { Mittelw. }\end{array}$ & Median & Min & Max & $\begin{array}{l}\text { Mittelw. } \\
\end{array}$ & Median & Min & Max \\
\hline $\begin{array}{l}\text { Anzahl Schülerinnen } \\
\text { und Schüler }\end{array}$ & 230,1 & 235 & 70 & 436 & 230,0 & 220 & 94 & 470 \\
$\begin{array}{l}\text { Anzahl Lehrkräfte } \\
\begin{array}{l}\text { Anzahl aufnehmender } \\
\text { Schulen }\end{array}\end{array}$ & 12,0 & 12 & 4 & 26 & 15,9 & 15 & 4 & 35 \\
& 6,9 & 6 & 3 & 16 & 6,8 & 6 & 2 & 16 \\
\hline
\end{tabular}

eine Gruppe erfahrener Lehrkräfte: In 2002 gaben 59 Lehrkräfte (83,1\%) an, seit mehr als zehn Jahren im Schuldienst tätig zu sein; in 2018 waren es 96 Lehrkräfte (90,6\%). Der Anteil an Befragten mit Schulleitungsfunktion beträgt 56,5\% in 2002 bzw. $73,8 \%$ in 2018 .

Die Schulen variieren deutlich in ihrer Größe (Schülerzahl; Größe des Kollegiums) und hinsichtlich der Anzahl an weiterführenden Schulen, auf welche die Kinder nach dem vierten Schuljahr wechseln (aufnehmende Schulen). Die Unterschiede zwischen den beiden Erhebungszeitpunkten sind jedoch gering (vgl. Tab. 1).

\subsection{Instrument}

Kernstück des Instruments ist eine Liste an Gestaltungsmaßnahmen, die sich auf die folgenden sechs Bereiche beziehen (in Klammern wird jeweils ein Beispielitem angegeben):

- sozio-emotionale Begleitung der Kinder (Mögliche Ängste/Sorgen hinsichtlich des Schulwechsels werden im Unterricht oder in Einzelgesprächen thematisiert.)

- unterrichtsbezogene Vorbereitung (Im Unterricht des vierten Schuljahrs werden der Schulwechsel und die damit verbundene Veränderungen thematisiert, z. B. als Thema im Deutschunterricht).

- diagnostisches Handeln im weiteren Sinne (Die GS-Lehrkräfte dokumentieren Lernstand und -entwicklung als Vorbereitung für die Übergangsempfehlung schriftlich, z. B. mit Profilbögen.)

- Elternberatung (Es finden über die rechtlich verbindlichen Informationsveranstaltungen hinausgehende weitere Beratungsangebote zum Schulwechsel statt.)

- Organisationsentwicklung (Es gibt ein (schriftlich fixiertes) Konzept zur Übergangsgestaltung.)

- Personalentwicklung (Die Grundschullehrkräfte nehmen an Fortbildungen zur Übergangsthematik teil)

Für jede Maßnahme wurde angegeben, ob diese aktuell praktiziert wird $(0=$ nein, $1=\mathrm{ja}$ ), wie wichtig sie für eine gelungene Übergangsgestaltung eingeschätzt $(1=$ gar nicht wichtig bis $4=$ sehr wichtig) und wie aufwändig die Durchführung erachtet wird ( $1=$ gar nicht schwierig bis $4=$ sehr schwierig). Die Maßnahmen wurden in Vorgängerstudien mit offenem Antwortformat entwickelt und den zuvor dargestellten Themenbereichen zugeordnet (siehe auch Tab. 2).

Neben diesen Informationen wurden Rahmendaten über die antwortende Person (Geschlecht, Berufserfahrung, Schulleitungsfunktion) und die Schule (Anzahl der 
Schülerinnen und Schüler, der Lehrkräfte, wichtiger aufnehmender Schulen) erhoben.

\subsection{Auswertungsverfahren}

Für die explorativen deskriptiven Analysen werden die Maßnahmen als Analyseeinheit genutzt. Pro Maßnahme wurden die Merkmale Verbreitungsgrad, Relevanz und Schwierigkeit aus den Angaben der Lehrkräfte generiert. Der Verbreitungsgrad entspricht dem Anteil der Schulen, in denen die Maßnahme aktuell praktiziert wird. Relevanz und Schwierigkeit wurden als Mittelwert über die jeweiligen Lehrkräfteeinschätzungen berechnet. (Rang)Korrelationen wurden berechnet für den Zusammenhang des Verbreitungsgrads in 2002 vs. 2018 sowie für den Zusammenhang von Verbreitungsgrad und Relevanz. Um zusätzlich den Einfluss der Schwierigkeit der Umsetzung zu berücksichtigen, wurde ein Regressionsmodell angepasst. Um spezifische und gemeinsame Varianzanteile zu bestimmen, wurde eine Kommunalitätenanalyse durchgeführt.

\section{Ergebnisse}

\subsection{Praktizierte Maßnahmen}

In Tab. 2 ist pro Maßnahme die prozentuale Häufigkeit notiert, mit der diese nach Auskunft der befragten Lehrkräfte aktuell an ihrer Schule umgesetzt wird. Die Werte variieren erheblich und liegen zwischen 98\% (informeller Austausch über Schülerinnen und Schüler; Abschlussfest feiern) und knapp 23\% (gemeinsame Fortbildung mit Lehrkräften der weiterführenden Schule). Den durchschnittlich höchsten Verbreitungsgrad erreichen die Maßnahmen der Elternberatung (86,3\%). Systematische Schulentwicklungsmaßnahmen im Sinne von Organisationsentwicklung $(47,4 \%)$ oder Personalentwicklung (35,0\%) finden vergleichsweise seltener statt. Schülerbezogene Maßnahmen, die auf den Unterricht der weiterführenden Schule bzw. auf soziale und emotionale Anpassungsleistungen vorbereiten, nehmen mit Blick auf den Verbreitungsgrad einen mittleren Platz ein (fachlich 70,0\%, sozial 71,4\%). Einen ähnlichen durchschnittlichen Verbreitungsgrad haben die hier abgefragten Maßnahmen, die das diagnostische Handeln der Lehrkräfte im weiteren Sinne vorbereiten (72,9\%). In allen Kategorien werden diejenigen Maßnahmen, die stufenübergreifend agieren, seltener praktiziert als Maßnahmen, die keine Kooperation erfordern (s. Tab. 2, Kennzeichnung mit ${ }^{\mathrm{a}}$ ).

\subsection{Zusammenhang von Relevanz und Verbreitungsgrad}

Es zeigt sich insgesamt ein sehr hoher Zusammenhang von Verbreitungsgrad und lehrerseitig eingeschätzter Relevanz (siehe Abb. 2). Die Rangkorrelation liegt bei $\mathrm{r}_{\text {spearman }}=0,906$.

Dennoch können verschiedene Maßnahmen identifiziert werden, bei denen die Rangplätze für Relevanz und Verbreitungsgrad um mehr als fünf Rangplätze aus- 
Tab. 2 Vergleich des Verbreitungsgrads der Maßnahmen in 2002 vs. 2018

\begin{tabular}{|c|c|c|c|}
\hline Themenbereich & Einzelmaßnahme (Kurzform) & 2002 & 2018 \\
\hline \multirow{6}{*}{$\begin{array}{l}\text { Elternberatung } \\
\mathrm{m}_{2002}=86,8 \\
\mathrm{~m}_{2018}=86,3\end{array}$} & Weitergabe von offiziellem Informationsmaterial & 91,4 & 94,9 \\
\hline & Regelmäßige Thematisierung in Elterngesprächen & 88,1 & 94,9 \\
\hline & Weitere Beratungsangebote & 88,6 & 93,8 \\
\hline & Elterninformation durch Schulleitung & 98,6 & 92,9 \\
\hline & Beratung für Eltern mit dem Kind & 78,6 & 82,1 \\
\hline & Erstellung von eigenem Informationsmaterial & 75,4 & 59,2 \\
\hline \multirow{6}{*}{$\begin{array}{l}\text { Diagnostisches } \\
\text { Handeln i. w. S. } \\
\mathrm{m}_{2002}=68,4 \\
\mathrm{~m}_{2018}=72,9\end{array}$} & Informeller Austausch der GS-LK über SuS & 97,0 & 98,0 \\
\hline & Erstellung von Profilbögen & n.e. & 93,7 \\
\hline & Informeller Austausch der GS-LK über WS & 89,6 & 87,4 \\
\hline & Teilnahme an Konferenzen an der $\mathrm{WS}^{\mathrm{a}}$ & 58,6 & 70,9 \\
\hline & Regelmäßige Anwesenheit an WS ${ }^{\mathrm{a}}$ & 69,6 & 51,8 \\
\hline & Unterrichtshospitation an $\mathrm{WS}^{\mathrm{a}}$ & 27,1 & 35,6 \\
\hline \multirow{6}{*}{$\begin{array}{l}\text { Sozio-emotionale } \\
\text { Begleitung der } \\
\text { Schülerinnen und } \\
\text { Schüler } \\
\mathrm{m}_{2002}=72,1 \\
\mathrm{~m}_{2018}=72,4\end{array}$} & Abschiedsfest feiern & 95,5 & 98,0 \\
\hline & Individuelle Sorgen besprechen & 91,0 & 95,9 \\
\hline & Vorbereitung auf soziale Herausforderungen & 84,8 & 74,5 \\
\hline & Kontakthalten mit Kindern auf WS nach dem Wechsel & 61,8 & 69,8 \\
\hline & Erkundung der $\mathrm{WS}^{\mathrm{a}}$ & 83,6 & 62,5 \\
\hline & $\begin{array}{l}\text { Außerunterrichtliche Aktivitäten gemeinsam mit Kindern } \\
\text { der WS }\end{array}$ & 15,6 & 27,6 \\
\hline \multirow{5}{*}{$\begin{array}{l}\text { Unterrichtsbezogene } \\
\text { Vorbereitung der } \\
\text { Schülerinnen und } \\
\text { Schüler } \\
\mathrm{m}_{2002}=65,7 \\
\mathrm{~m}_{2018}=70,0\end{array}$} & Übergang als Unterrichtsthema & 91,2 & 87,5 \\
\hline & Höhere Anzahl unterrichtender Lehrkräfte & 69,2 & 85,6 \\
\hline & Allmähliche Anpassung an Anforderungen der WS & 86,2 & 77,0 \\
\hline & Allmähliche Anpassung an Methoden der WS & 72,3 & 64,6 \\
\hline & Unterrichtliche Aktivitäten mit Kindern der WS ${ }^{a}$ & 9,4 & 35,4 \\
\hline \multirow{8}{*}{$\begin{array}{l}\text { Organisations- } \\
\text { entwicklung } \\
\mathrm{m}_{2002}=41,2 \\
\mathrm{~m}_{2018}=47,4\end{array}$} & Individuelle LK halten Kontakt zu wichtigen WS ${ }^{\mathrm{a}}$ & 95,7 & 90,6 \\
\hline & Schriftliches Konzept zum Übergang & 50,7 & 59,8 \\
\hline & Fester Ansprechpartner für $\mathrm{WS}^{\mathrm{a}}$ & 51,5 & 58,1 \\
\hline & $\begin{array}{l}\text { Weitergabe von Informationen über Lernstand/-entwick- } \\
\text { lung der SuS an WS }\end{array}$ & 31,9 & 47,1 \\
\hline & Absprachen Methoden \& Inhalte mit WS ${ }^{\mathrm{a}}$ & 37,7 & 37,1 \\
\hline & Informelle AGs mit Lehrkräften der WS ${ }^{\mathrm{a}}$ & 14,9 & 30,2 \\
\hline & Kooperation in stufenübergreifendem Schulnetzwerk ${ }^{\mathrm{a}}$ & n.e. & 28,8 \\
\hline & Interne $A G$ an eigener Schule & 6,1 & 27,0 \\
\hline \multirow{3}{*}{$\begin{array}{l}\text { Personalentwicklung } \\
\mathrm{m}_{2002}=26,2 \\
\mathrm{~m}_{2018}=35,0\end{array}$} & Pädagogische Konferenz zum Übergang & 34,3 & 45,8 \\
\hline & Teilnahme an Fortbildungen zum Übergang & 35,8 & 36,5 \\
\hline & Gemeinsame Fortbildungen mit WS-LK ${ }^{\mathrm{a}}$ & 8,6 & 22,7 \\
\hline
\end{tabular}

Tabellenwerte sind prozentuale Häufigkeiten. Die Reihung erfolgt nach dem Verbreitungsgrad in 2018. Mit ${ }^{a}$ markierte Maßnahmen erfordern schulstufenübergreifende Kooperation 


\begin{abstract}
Abb. 2 Verbreitungsgrad und wahrgenommene Relevanz der Gestaltungsmaßnahmen im Vergleich. (Messwerte sind Rangplätze von Verbreitungsgrad und Relevanz. Punkte oberhalb (unterhalb) der Diagonalen $=$ Maßnahmen, die in Relation zum Verbreitungsgrad eher als relevant (irrelevant) erachtet werden)
\end{abstract}

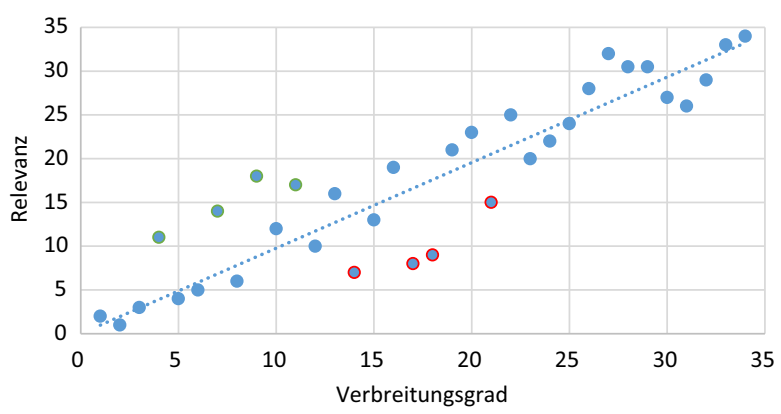

einanderliegen. Einerseits sind dies Maßnahmen, die trotz eher geringer Verbreitung als relevant für eine unterstützende Übergangsgestaltung erachtet werden. Diese vier Maßnahmen beziehen sich alle auf stufenübergreifende Kooperationen (Absprachen Methoden und Inhalten; Hospitation; Schulnetzwerke; Weitergabe von Informationen). Hingegen werden ebenfalls vier Maßnahmen trotz gering wahrgenommener Relevanz mit mittlerer Häufigkeit praktiziert (Anpassung von Unterrichtsmethoden; Anpassung von Leistungsanforderungen; Kontakthalten mit Kindern nach Wechsel; Erstellung eigener Informationsmaterialien).

\subsection{Vorhersage des Verbreitungsgrades durch Relevanz und Schwierigkeit}

Mittels Regressionsanalyse wird der Verbreitungsgrad der Maßnahmen vorhergesagt über die von den Lehrkräften eingeschätzte (mittlere) Relevanz und Schwierigkeit (siehe Tab. 3).

Es zeigt sich einerseits ein positiver Zusammenhang des Verbreitungsgrads mit der Relevanz und andererseits ein negativer Zusammenhang mit der Schwierigkeit. Dabei ist die Schwierigkeit der Durchführung einer Maßnahme in vergleichbarem Maße prädiktiv für ihre Umsetzung wie die wahrgenommene Relevanz: Die Anteile der spezifisch durch die Schwierigkeit erklärten Varianz fallen ähnlich groß aus wie die spezifische Varianz für die Relevanz und ebenso sind die Regressionsgewichte für Schwierigkeit dem Betrage nach von vergleichbarer Größe.

\subsection{Vergleich praktizierter Maßnahmen 2002 vs. 2018}

Maßnahmen, die in 2002 an vielen Grundschulen praktiziert wurden, werden auch in 2018 noch häufig praktiziert $\left(\mathrm{r}_{\text {Pearson }}=0,940\right)$. Die grafische Darstellung (siehe Abb. 3)

Tab. 3 Ergebnisse der Regressionsanalyse zur Vorhersage des Verbreitungsgrades

\begin{tabular}{lll}
\hline Regressionsgewicht (beta) & Relevanz & 0,480 \\
& Schwierigkeit & $-0,518$ \\
Varianzaufklärung & $\mathrm{R}^{2}$ Gesamt & $88,9 \%$ \\
Varianzverteilung & Spezifisch Relevanz & $9,9 \%$ \\
& Spezifisch Schwierigkeit & $11,5 \%$ \\
& Konfundiert & $78,6 \%$ \\
\hline
\end{tabular}


Abb. 3 Verbreitungsgrad der Gestaltungsmaßnahmen im zeitlichen Vergleich (2002 vs. 2018). (Punkte oberhalb (unterhalb) der Diagonalen $=$ Maßnahmen, die in 2018 häufiger (seltener) praktiziert wurden als in 2002)

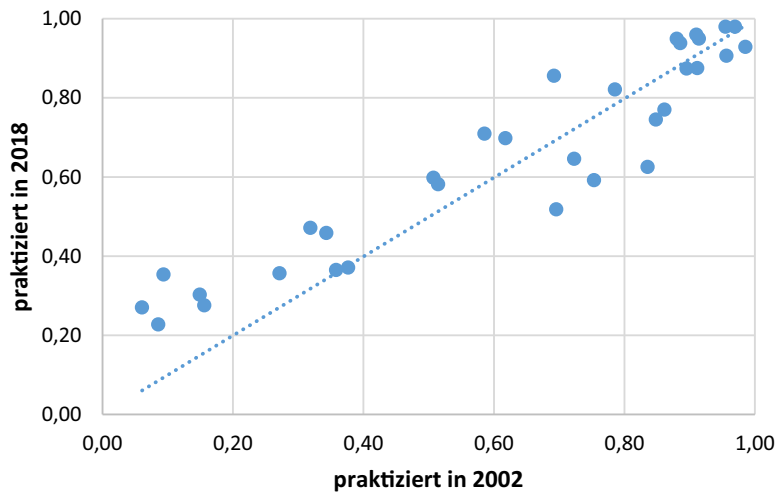

zeigt insbesondere, dass die in 2002 selten praktizierten Maßnahmen zwar auch 2018 immer noch am unteren Ende der Rangreihe stehen, in ihrer Häufigkeit jedoch zugenommen haben (Datenpunkte liegen oberhalb der Referenzlinie). Insgesamt stehen 21 Maßnahmen, die häufiger praktiziert werden 11 Maßnahmen mit einer geringeren Nennungshäufigkeit gegenüber. Die mittlere Gesamtanzahl praktizierter Maßnahmen hat pro Schule von 20,3 auf 22,7 zugenommen.

In 2002 wurden vier Maßnahmen an mehr als 95\% der Schulen durchgeführt. Dies sind im Einzelnen die Informationsveranstaltung für Eltern durch die Schulleitung, der informelle Austausch über einzelne Schülerinnen und Schüler innerhalb des Kollegiums, das Kontakthalten zu wichtigen aufnehmenden Schulen sowie das Feiern eines Abschlussfestes. Alle vier Maßnahmen werden auch in 2018 sehr häufig praktiziert, allerdings mit leicht geringeren Häufigkeiten für Informationsveranstaltung und Kontakthalten. Daneben werden eine schülerorientierte sozio-emotionale Maßnahme (Eingehen auf individuelle Sorgen der Kinder) und zwei Maßnahmen aus dem Bereich der Elternberatung (Weitergabe von offiziellem Informationsmaterial und regelmäßige Thematisierung in Elterngesprächen) an mindestens $95 \%$ der teilnehmenden Schulen praktiziert.

Am unteren Ende der Rangliste lagen 2002 die Etablierung einer schulinternen AG zum Übergang, die Teilnahme an Fortbildungen gemeinsam mit den Lehrkräften der weiterführenden Schulen, sowie gemeinsame stufenübergreifende, unterrichtliche Aktivitäten für die Kinder des vierten und fünften Schuljahres. Diese Maßnahmen wurden zum ersten Befragungszeitpunkt an weniger als $15 \%$ der teilnehmenden Schulen praktiziert. Auch 2018 bilden die gemeinsamen Fortbildungen und die schulinternen AGs das Schlusslicht in der Rangreihe. Allerdings haben sich die Anteile an Schulen, die angeben, dies zu praktizieren, mit $23 \%$ respektive $27 \%$ mehr als verdoppelt. An drittletzter Stelle finden sich in 2018 stufenübergreifende außerunterrichtliche Aktivitäten für die Kinder (28\%), während stufenübergreifende unterrichtliche Aktivitäten von immerhin $35 \%$ der Befragten als eine aktuell praktizierte Maßnahme der Übergangsgestaltung angegeben werden.

Eine um 15 Prozentpunkte häufigere Durchführung ergibt sich für folgende Maßnahmen: An vorderster Stelle stehen zwei in 2002 besonders selten praktizierte Kooperationsmaßnahmen (stufenübergreifende, unterrichtliche Aktivitäten 
(9,4\%:35,4\%) und schulinterne AGs (6,1\%:27,0\%)). Zwei weitere stufenübergreifende Kooperationsformen haben besonders hohe Zuwächse zu verzeichnen (stufenübergreifende informelle AGs (14,9\%:30,2\%) und Weitergabe von Infos über

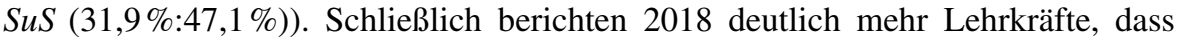
an ihren Schulen versucht wird, die Anzahl unterrichtender Lehrkräfte im vierten Schuljahr bereits zu erhöhen, um sich dem Fachlehrerprinzip der weiterführenden Schule anzunähern $(69,2 \%: 85,6 \%)$.

Andererseits werden drei Maßnahmen in 2018 seltener als 15 Jahre zuvor praktiziert und verzeichnen eine um 15 Prozentpunkte geringere Nennungshäufigkeit (Erkundung der weiterführenden Schulen (83,6\%:62,5\%); regelmäßige Anwesenheit an weiterführenden Schulen (69,6\%:51,8\%), eigenes Informationsmaterial über die weiterführenden Schulen $(75,4 \%: 59,2 \%))$.

\section{Diskussion}

Basierend auf dem Übergangsqualitätsmodell von van Ophuysen und Harazd (2014) wurden sechs Bereiche benannt, in denen Maßnahmen der Übergangsgestaltung der Grundschule verortet werden können. Der Verbreitungsgrad der Maßnahmen kann durch die mittlere Schwierigkeits- und Relevanzeinschätzung gut vorhergesagt werden. Erwartungskonform werden als relevant eingeschätzte Maßnahmen häufiger umgesetzt; Maßnahmen, deren Umsetzung als schwierig eingeschätzt wird, sind hingegen weniger verbreitet. Insgesamt werden in 2018 etwas mehr Maßnahmen an den Schulen praktiziert als im Jahr 2002, wobei gerade bei den Aktivitäten, die die Kooperation mit weiterführenden Schulen erfordern, in 2018 zumeist eine Zunahme zu verzeichnen ist. Insgesamt ändert sich die Rangfolge der Maßnahmen mit Blick auf ihre Verbreitung jedoch kaum, und kooperative Maßnahmen stehen weiterhin eher am unteren Ende der Rangliste. Bevor die Befunde für die verschiedenen Inhaltsbereiche diskutiert werden, sollen kurz methodische Aspekte der Studie kritisch reflektiert werden.

\subsection{Forschungsmethodische Reflexion}

Insgesamt liefert die hier vorliegende Datenbasis einen ersten Einblick, wie an nordrheinwestfälischen Grundschulen der Übergang zur weiterführenden Schule vorbereitet und begleitet wird. Das hier genutzte, standardisierte Erhebungsformat birgt das Problem, dass das individuelle Verständnis bestimmter Maßnahmen möglicherweise nicht einheitlich war. Begriffe wie ,informelle Arbeitsgruppen“ oder „Kontakt halten“" wurden vielleicht unterschiedlich verstanden. Auch liefert die Antwort, dass eine Maßnahme praktiziert wird, keinen Hinweis auf die Durchführungsqualität. Hier wären Interviews oder offene Befragungen erforderlich, über die aber andererseits weder die Vielzahl an Schulen noch die Breite an Maßnahmen hätte erfasst werden können.

In der statistischen Analyse wurden die Maßnahmen als Analyseeinheit genutzt, die jeweils durch die Merkmale Verbreitungsgrad, Relevanz und Schwierigkeit charakterisiert sind. Die erhobenen Maßnahmen stellen ihrerseits eine Stichprobe von 
Aktivitäten dar, die an Schulen durchgeführt werden. Damit ist die Aussagekraft der Studie auch von der Qualität der Maßnahmenauswahl abhängig. Diese wurden im Rahmen der Vorläuferstudie aus 2002 auf Basis von qualitativen Vorerhebungen entwickelt. Die offene Abfrage weiterer Maßnahmen ergab keine weiteren Maßnahmen, die von mehreren Personen genannt wurde, so dass der Maßnahmenpool die Aktivitäten der Schulen scheinbar gut abbildet.

Mit Blick auf die Stichprobe ist die Aussagekraft der vorliegenden Daten einerseits relativ hoch, da die Fragebögen weitestgehend von Personen beantwortet wurden, die mit vieljähriger Berufserfahrung entweder als Teil der Schulleitung oder als verantwortliche Lehrkraft eines vierten Schuljahres tätig sind. Die antwortenden Lehrkräfte sind Teil einer Zufallsstichprobe, die auf Basis der Schulliste in NRW gezogen wurde. Jedoch sind auch Einschränkungen zu beachten. Wenngleich der Rücklauf für eine nicht weiter in ein Projekt eingebundene ad-hoc Befragung angemessen erscheint, sind dennoch Verzerrungstendenzen nicht auszuschließen. Möglicherweise fühlten sich gerade solche Schulen von der Befragung angesprochen, die besonders aktiv in der Übergangsgestaltung sind, wodurch insgesamt eine Überschätzung des Verbreitungsgrads der Maßnahmen möglich ist.

\subsection{Gestaltung des Übergangs}

\subsubsection{Fachliche und affektive Unterstützung der Schülerinnen und Schüler}

Die von uns abgefragten Maßnahmen im Bereich der fachlichen und sozio-emotionalen Vorbereitung der Kinder auf den Übergang werden an den meisten Schulen praktiziert. Ein Abschiedsfest zu feiern und individuelle Sorgen zu besprechen gehören an den im Jahr 2018 befragten Schulen ebenso zum Standardrepertoire wie die Bearbeitung des Übergangs als Unterrichtsthema oder die Hinführung auf das Fachlehrkräfte-Konzept durch den verstärkten Einsatz von mehreren Lehrkräften in den Klassen des vierten Jahrgangs. An weniger Schulen wird hingegen eine bewusste Anpassung der Lernmethoden an die Methoden der weiterführenden Schulen praktiziert. Diesbezüglich äußerte beispielsweise eine Lehrkraft in einem offenen Kommentarfeld: „Ärgerlich ist die Vorstellung, dass sich Grundschule an die WS anpassen soll. Kinder werden individuell gefördert und gefordert. Warum soll dann an die weiterführende Schule angepasst werden?“. Und eine andere Lehrkraft merkte an: ,Warum soll sich der Unterrichtsstil der Grundschulen an den, der weiterführenden Schulen anpassen? Warum wird das kooperative Arbeiten, die Arbeitstechniken der GS an den weiterführenden Schulen nicht auch übernommen?“.

Beim Vergleich der Daten von 2018 mit den Informationen aus 2002 zeigen sich lediglich geringfügige Veränderungen. Einzig die beiden Maßnahmen, die die explizite Arbeit mit Kindern beider Schulstufen implizieren (unterrichtliche und außerunterrichtliche Aktivitäten gemeinsam mit den Kindern der weiterführenden Schulen), haben eine nennenswerte Zunahme zu verzeichnen, werden aber auch in 2018 nur bei etwa einem Drittel der befragten Schulen praktiziert. 


\subsubsection{Diagnostik und Beratung}

Den insgesamt höchsten Verbreitungsgrad haben die von uns abgefragten Maßnahmen im Kontext der Elternarbeit. Dies erscheint plausibel, da hier die einzigen Maßnahmen enthalten sind, die rechtlich verbindlich sind (Information durch Schulleitung) bzw. ministeriell unterstützt werden (Weitergabe von offiziellem Informationsmaterial). Verwunderlich ist hier eher, dass diese Maßnahmen nicht von allen Schulen angeführt werden. Veränderungen über die letzten 15 Jahre sind in diesem Bereich nicht erkennbar.

Maßnahmen, die das diagnostische Handeln im weiteren Sinne unterstützen, indem sie beispielsweise Multiperspektivität fördern, eine systematische Dokumentation von Schülerinformation implizieren und insbesondere das Wissen über die Anforderungen und Bedingungen an den weiterführenden Schulen erhöhen, werden ebenfalls recht häufig praktiziert und haben in den letzten 15 Jahren noch zugenommen. Gerade die Teilnahme an (Erprobungsstufen-)Konferenzen findet häufiger statt als vor 15 Jahren und wurde in 2018 an über zwei Drittel der befragten Schulen praktiziert. Die Lehrkräfte können sich so über die Entwicklung ,ihrer“ Kinder informieren und ein Feedback zu ihrer Übergangsempfehlung erhalten. Wenngleich es keine explizite rechtliche Verankerung gibt, werden auch in der aktuellen KMKEmpfehlung zum Grundschulübergang gemeinsame Konferenzen als wünschenswerte Praxis angeführt (KMK 2015).

\subsubsection{Personal-/Organisationsentwicklung}

Vergleichsweise selten werden Maßnahmen aus dem Bereich der Organisationsentwicklung praktiziert. Hier wurden neben Maßnahmen an der Grundschule (z. B. schriftliches Konzept zur Übergangsgestaltung) vor allem solche Aktivitäten erfragt, die in Kooperation mit den weiterführenden Schulen erfolgen (z. B. Absprachen über Methoden und Inhalte). Insgesamt ist eine leichte Zunahme institutionalisierter Maßnahmen zu erkennen.

Auch Maßnahmen der Personalentwicklung wie die Teilnahme an Fort- und Weiterbildungen sowie pädagogischen Konferenzen zum Thema Übergang wurden in 2018 deutlich häufiger berichtet als vor 15 Jahren. Insgesamt werden diese Aktivitäten jedoch eher selten praktiziert. Gerade die stufenübergreifende Fortbildung gemeinsam mit Lehrkräften der weiterführenden Schulen hat nur eine vergleichsweise geringe Verbreitung.

Da der Übergang in aktuellen Fortbildungsangeboten für Lehrkräfte allenfalls vereinzelt thematisiert wird (vgl. https://suche.lehrerfortbildung.schulministerium. nrw.de/), mag hier aber auch ein geringes Angebot (mit)ursächlich sein. Um eine Erhöhung der Durchführungshäufigkeit dieser Maßnahmen zu erzielen, könnte entsprechend ein einschlägiges Fortbildungsangebot der Bezirksregierungen oder der Regionalen Bildungsbüros hilfreich sein. Gleichzeitig ist aber relativierend anzumerken, dass von Themen wie der schulischen Inklusion oder der Digitalisierung aktuell ein starker Handlungsdruck ausgeht, so dass Fort- und Weiterbildungen eher in diesen Bereichen nachgefragt werden. 


\subsubsection{Stufenübergreifende Kooperation}

Das Übergangsqualitätsmodell impliziert einen (positiven) Einfluss von institutionalisierter Kooperation und Vernetzung über die Schulstufen hinweg auf die relevanten Prozessmerkmale an den Grundschulen. Immerhin knapp 30\% der befragten Schulen gaben an, in einem stufenübergreifenden Netzwerk eingebunden zu sein. Diese Maßnahme wurde erst 2018 in den Maßnahmenkatalog aufgenommen. Erste weiterführende Analysen zeigen, dass stufenübergreifende Aktivitäten in Schulen, die Teil eines Netzwerks sind, in der Tat häufiger praktiziert werden: in Netzwerkschulen sind die Anpassung des Unterrichts an die Methoden der weiterführenden Schulen und gemeinsame unterrichtliche Aktivitäten weiter verbreitet. Die befragten Lehrkräfte von Netzwerkschulen gaben häufiger als die anderen Lehrkräfte an, die weiterführenden Schulen zu erkunden und auch nach dem Übergang den Kontakt zu den Kindern zu halten. Durch vermehrte Hospitation und Anwesenheit an den weiterführenden Schulen kennen die Lehrkräfte die Methoden und Anforderungen vor Ort, was auch zu einer besseren Selektionsentscheidung beitragen kann. Diese Befunde stehen in Übereinstimmung mit den positiv wahrgenommenen Konsequenzen der Netzwerkarbeit in einer Lehrkräftebefragung im Kontext des Projektes Schulen im Team (Sartory 2016).

Obwohl stufenübergreifende Maßnahmen häufiger als vor 15 Jahren praktiziert werden, ergibt sich gerade für sie immer noch eine hohe Rangplatzdifferenz zwischen Verbreitungsgrad und Relevanz (siehe zum Vergleich van Ophuysen 2005). Die Regressionsanalyse verdeutlicht, dass die als eher hoch wahrgenommene Schwierigkeit dieser Maßnahmen als (statistische) Begründung für die Diskrepanz herangezogen werden kann. Wenngleich der korrelative Befund kein valider Hinweis auf Kausalität ist, ist es durchaus plausibel anzunehmen, dass der Verbreitungsgrad gewünschter Maßnahmen durch verstärkte Unterstützung bei deren Etablierung erhöht werden könnte. So ist aus der schulischen Implementationsforschung bekannt, dass Innovationen, dann umgesetzt werden, wenn die Beteiligten einen hohen Bedarf sehen und die benötigten Fähigkeiten, Ressourcen und Unterstützungsmöglichkeiten vorhanden sind (Altrichter und Wiesinger 2005). Neben dem Angebot von Fortbildungen könnte die verstärkte Kommunikation von Praxistipps und konkreten Handreichungen zielführend sein, um verantwortlichen Personen die Einführung als relevant erachteter Maßnahmen zu erleichtern. Eine solche Unterstützung kann aber eben auch gezielt in Schulnetzwerken erfolgen. Für gelingende Netzwerkarbeit erscheint dabei ein systematisches Übergangsmanagement auf kommunaler Ebene als eine förderliche, wenn nicht sogar erforderliche Voraussetzung (Sartory et al. 2018). Durch solche Netzwerke wären vermutlich weitere positive Effekte zu erwarten; beispielsweise Ideenaustausch und -transfer, Professionalisierung der Lehrkräfte durch gemeinsame Reflexionsprozesse oder die Bündelung von Kompetenzen zur gemeinsamen Zielerreichung (Jungermann et al. 2018).

\subsection{Fazit}

Mit der vorliegenden Studie wurden insbesondere der Verbreitungsgrad und die Veränderung für eine Auswahl an Maßnahmen untersucht, die sich im Rahmen des 
Übergangsqualitätsmodells auf Input- und Prozessebene verorten lassen. Im Sinne von Interventions- und Evaluationsforschung können in zukünftigen Projekten Umsetzungsbedingungen und die Wirksamkeit einzelner Maßnahmen oder Maßnahmenbündel analysiert werden. Doch bereits jetzt kann für die (Grund)Schulpraxis die Systematisierung der Maßnahmenbereiche ebenso wie ein Katalog der einzelnen Maßnahmen als hilfreiche Orientierung dienen. So können im Rahmen von Schulentwicklungsprozessen auf dieser Basis bestehende Übergangskonzepte reflektiert, Zielsetzungen hinterfragt und ggf. Ideen für weitere Maßnahmen abgeleitet werden.

Funding Open Access funding enabled and organized by Projekt DEAL.

Open Access Dieser Artikel wird unter der Creative Commons Namensnennung 4.0 International Lizenz veröffentlicht, welche die Nutzung, Vervielfältigung, Bearbeitung, Verbreitung und Wiedergabe in jeglichem Medium und Format erlaubt, sofern Sie den/die ursprünglichen Autor(en) und die Quelle ordnungsgemäß nennen, einen Link zur Creative Commons Lizenz beifügen und angeben, ob Änderungen vorgenommen wurden.

Die in diesem Artikel enthaltenen Bilder und sonstiges Drittmaterial unterliegen ebenfalls der genannten Creative Commons Lizenz, sofern sich aus der Abbildungslegende nichts anderes ergibt. Sofern das betreffende Material nicht unter der genannten Creative Commons Lizenz steht und die betreffende Handlung nicht nach gesetzlichen Vorschriften erlaubt ist, ist für die oben aufgeführten Weiterverwendungen des Materials die Einwilligung des jeweiligen Rechteinhabers einzuholen.

Weitere Details zur Lizenz entnehmen Sie bitte der Lizenzinformation auf http://creativecommons.org/ licenses/by/4.0/deed.de.

\section{Literatur}

Altrichter, H., \& Wiesinger, S. (2005). Implementation von Schulinnovationen - aktuelle Hoffnungen und Forschungswissen. Journal für Schulentwicklung, 9(4), 28-36.

Autorengruppe Bildungsberichterstattung (2016). Bildung in Deutschland 2016. Ein indikatorengestützter Bericht mit einer Analyse zu Bildung und Migration. Bielefeld: wbv.

Baumert, J., Maaz, K., \& Trautwein, U. (Hrsg.). (2010). Bildungsentscheidungen. Wiesbaden: VS.

Baumert, J., Stanat, P., \& Watermann, R. (Hrsg.). (2006). Herkunftsbedingte Disparitäten im Bildungswesen: Differenzielle Bildungsprozesse und Probleme der Verteilungsgerechtigkeit. Vertiefende Analysen im Rahmen von PISA 2000. Wiesbaden: VS.

Behörde für Schule und Berufsbildung (2011). Bildungsbericht. Hamburg: Behörde für Schule und Berufsbildung.

Bos, W., \& Gröhlich, C. (Hrsg.). (2011). KESS 8 - Kompetenzen und Einstellungen von Schülerinnen und Schülern am Ende der Jahrgangsstufe 8. Münster: Waxmann.

Büchner, P., \& Koch, K. (2001). Der Übergang aus Kinder- und Elternsicht. Von der Grundschule in die Sekundarstufe, Bd. 1. Opladen: Leske + Budrich.

Dalin, P., Rolff, H.-G., \& Buchen, H. (1995). Institutioneller Schulentwicklungsprozess. Bönen: Kettler.

Ditton, H. (2000). Qualitätskontrolle und Qualitätssicherung in Schule und Unterricht. Ein Überblick zum Stand der empirischen Forschung. Zeitschrift für Pädagogik, Beiheft, 41, 79-92.

Fend, H. (1986). Gute Schulen - schlechte Schulen. Die einzelne Schule als pädagogische Handlungseinheit. Die deutsche Schule, 78(3), 275-293.

Hagenauer, G. (2011). Lernfreude in der Schule. Münster: Waxmann.

Hildebrandt, J. (2016). Das Erleben von Herausforderung und Bedrohung am Grundschulübergang. Analysen zu Validität, Leistungsentwicklung und Ressourcen. Dissertation. Berlin: Freie Universität Berlin. https://www.fachportal-paedagogik.de/literatur/vollanzeige.html?FId=1113086\#vollanzeige. Zugegriffen: 2. Juli 2019.

Jonkmann, K., Maaz, K., Neumann, M., \& Gresch, C. (2010). Übergangsquoten und Zusammenhänge zu familiärem Hintergrund und schulischen Leistungen: Deskriptive Befunde. In K. Maaz, J. Baumert, C. Gresch \& N. McElvany (Hrsg.), Der Übergang von der Grundschule in die weiterführende Schu- 
le. Leistungsgerechtigkeit und regionale, soziale und ethnisch-kulturelle Disparitäten (S. 255-284). Bonn: BMBF.

Jungermann, A., Pfänder, H., \& Berkemeyer, N. (2018). Schulische Vernetzung in der Praxis. Wie Schulen Unterricht gemeinsam entwickeln können. Münster: Waxmann.

KMK (2015). Empfehlungen zur Arbeit in der Grundschule. Beschluss der Kultusministerkonferenz vom 02.07.1970 i.d. F. vom 11.06.2015

Knoppick, H., Becker, M., Neumann, M., Maaz, K., \& Baumert, J. (2016). Das subjektive Erleben des Übergangs in die weiterführende Schule: Die Bedeutung der Antizipation für die Bewältigung dieses kritischen Lebensereignisses. Zeitschrift für Entwicklungspsychologie und Pädagogische Psychologie, 48(3), 129-143.

Kurtz, T., Watermann, R., Klingebiel, F., \& Szczesny, M. (2010). Das emotionale Erleben des bevorstehenden Grundschulübergangs und die Rolle der elterlichen Unterstützung. In K. Maaz, J. Baumert, C. Gresch \& N. McElvany (Hrsg.), Der Übergang von der Grundschule in die weiterführende Schule. Leistungsgerechtigkeit und regionale, soziale und ethnisch-kulturelle Disparitäten (S. 333-355). Bonn: BMBF.

Lazarus, R. S., \& Folkman, S. (1987). Transactional theory and research on emotions and coping. European Journal of Personality, 1(3), 141-169.

Leffelsend, S., \& Harazd, B. (2003). Konstruktion und Validierung eines Fragebogens zur Erfassung der affektiven Bewertung des Übergangs von der Grundschule zur weiterführenden Schule. In E. J. Brunner, P. Noack, G. Scholz \& I. Scholl (Hrsg.), Diagnose und Intervention in schulischen Handlungsfeldern (S. 141-158). Münster: Waxmann.

Leffelsend, S., \& Harazd, B. (2004). Erwartungen an die weiterführende Schule. Empirische Untersuchung zu den Übergangserwartungen von Grundschülern. Empirische Pädagogik, 18(2), 252-272.

Lintorf, K., van Ophuysen, S., \& Behrmann, L. (2016). Diagnostik im Lehrerberuf. In M. Rothland (Hrsg.), Beruf Lehrer/Lehrerin. Ein Studienbuch (S. 187-203). Münster: Waxmann.

Lohaus, A., Vierhaus, M., \& Ball, J. (2005). Der Wechsel zur weiterführenden Schule. Generelle und differenzielle Effekte auf Stresserleben und psychische Anpassung. In S. R. Schilling, J. R. Sparfeld \& S. Pruisken (Hrsg.), Aktuelle Aspekte pädagogisch-psychologischer Forschung (S. 87-110). Münster: Waxmann.

Maaz, K., \& Nagy, G. (2010). Der Übergang von der Grundschule in die weiterführenden Schulen des Sekundarschulsystems: Definition, Spezifikation und Quantifizierung primärer und sekundärer Herkunftseffekte. In J. Baumert, K. Maaz \& U. Trautwein (Hrsg.), Bildungsentscheidungen (S. 153-182). Wiesbaden: VS.

Maaz, K., Trautwein, U., Lüdtke, O., \& Baumert, J. (2008). Educational transitions and differential learning environments: How explicit between-school tracking contributes to social inequality in educational outcomes. Child Development Perspectives, 2(2), 99-106.

Ministerium für Schule und Bildung des Landes NRW (2019). Statistik-Telegramm 2018/19. Schuleckdaten 2018/19. Düsseldorf. https:/www.schulministerium.nrw.de/sites/default/files/documents/ StatTelegramm2018.pdf. Zugegriffen: 7. Aug. 2020.

van Ophuysen, S. (2005). Gestaltungsmaßnahmen zum Übergang von der Grundschule zur Weiterführenden Schule. In H. G. Holtappels \& K. Höhmann (Hrsg.), Schulentwicklung und Schulwirksamkeit (S. 141-152). Weinheim: Juventa.

van Ophuysen, S. (2006). Übergangserwartungen und der Start in der neuen Schule. In R. Hinz (Hrsg.), Professionelles Handeln in der Grundschule. Entwicklungslinien und Forschungsbefunde (S. 97-105). Baltmannsweiler: Schneider-Verlag Hohengehren.

van Ophuysen, S. (2013). Der Grundschulübergang aus der Perspektive der Schülerinnen und Schüler Befunde aus quantitativen Studien. In N. Berkemeyer, S.-I. Beutel, H. Järvinen \& S. van Ophuysen (Hrsg.), Übergänge bilden - Lernen in der Grund- und weiterführenden Schule (S. 98-121). Neuwied: Wolters Kluwer.

van Ophuysen, S. (2018). Der Übergang von der Grundschule auf die weiterführende Schule: Erleben und Entwicklung der Kinder. Theorie und Forschungsstand. In R. Porsch (Hrsg.), Der Übergang von der Grundschule auf weiterführende Schulen. Grundlagen für die Lehrerausbildung und Praxis (S. 115-137). Münster: utb.

van Ophuysen, S., \& Harazd, B. (2011). Der Übergang von der Grundschule zur weiterführenden SchuleGestaltung, Beratung, Diagnostik. Handreichung des Programms „SINUS an Grundschulen“. Kiel: IPN-Materialien.

van Ophuysen, S., \& Harazd, B. (2014). Der Übergang von der Grundschule zur weiterführenden Schule aus der Perspektive der Schulqualität: ein Übergangsqualitätsmodell. In M. Pfeifer (Hrsg.), Schulqualität und Schulentwicklung - Theorien, Analysen und Potenziale (S. 73-92). Münster: Waxmann. 
van Ophuysen, S., Lintorf, K., \& Harazd, B. (2013). Zur Qualität professioneller pädagogischer Diagnostik im Schulalltag - Forschungsbefunde und -desiderate. In K. Schwippert, M. Bonsen \& N. Berkemeyer (Hrsg.), Schul- und Bildungsforschung. Diskussionen, Befunde und Perspektiven (S. 187-202). Münster: Waxmann.

Porsch, R. (2015). Pädagogische Maßnahmen, Kooperationsformen und Herausforderungen an Ganztagsgymnasien im Übergang nach der Grundschule. In H. Wendt \& W. Bos (Hrsg.), Auf dem Weg zum Ganztagsgymnasium. Erste Ergebnisse der wissenschaftichen Begleitforschung zum Projekt Ganz In (S. 201-224). Münster: Waxmann.

Porsch, R. (Hrsg.). (2018). Der Übergang von der Grundschule auf weiterführende Schulen. Grundlagen für die Lehrerausbildung, Fortbildung und Praxis. Stuttgart: UTB.

Sartory, K. (2016). Lehrerkooperation am Übergang von der Grund- zur weiterführenden Schule. In R. Strietholt, W. Bos, H.-G. Holtappels \& N. McElvany (Hrsg.), Jahrbuch der Schulentwicklung (Bd. 19, S. 135-160). Weinheim: Juventa.

Sartory, K., Jungermann, A., Hoeft, M., \& Bos, W. (2018). Kommunales Übergangsmanagement in der Praxis. Wie Kommunen und Schulen Übergänge gemeinsam gestalten können. Münster: Waxmann.

Scheerens, J. (1990). School effectiveness and the development of process indicators of school functioning. School Effectiveness and School Improvement, 1, 61-80.

Schürer, S., Harazd, B., \& van Ophuysen, S. (2006). Übergangsgestaltung durch schulstufenübergreifende Lehrerkooperation. In R. Hinz (Hrsg.), Professionelles Handeln in der Grundschule. Entwicklungslinien und Forschungsbefunde (S. 90-96). Baltmannsweiler: Schneider-Verlag Hohengehren.

Sirsch, U. (2000). Probleme beim Schulwechsel. Die subjektive Bedeutung des bevorstehenden Wechsels von der Grundschule in die weiterführende Schule. Münster: Waxmann. 